\title{
Variation of the line profile moments for stars pulsating in distorted oblique non-radial modes
}

\begin{abstract}
O. Kochukhov
Department of Astronomy and Space Physics, Uppsala University, 75120 Uppsala, Sweden

e-mail: oleg@astro.uu.se

Received 3 January 2005 / Accepted 21 March 2005

Abstract. We derive expressions and develop a numerical technique for the analysis of the line profile moment variations for stars pulsating in oblique non-radial modes. This represents an extension of the widely used spectroscopic moment mode identification method to the oblique distorted pulsations observed in rapidly oscillating Ap stars. We demonstrate that a nonaxisymmetric superposition of the pulsation and rotation velocity fields results in a qualitatively new behaviour of some of the line profile characteristics. It is found that for the majority of roAp stars the second moment varies with the pulsation frequency rather than with its first harmonic even for axisymmetric modes. We also identify pulsation observables which do not change during pulsation cycle but are modulated by the stellar rotation and can contribute to the variability of the stellar spectra averaged over many pulsation cycles. As an illustration of the new version of the moment technique, we compute rotational modulation of the pulsational changes of the line profile moments for the oblique axisymmetric dipolar pulsation modes with different parameters. It is also shown that a distortion of the oblique dipolar modes predicted by the recent theoretical studies of the stellar magneto-acoustic oscillations can be readily diagnosed through the moment analysis. In particular, the shape of the pulsation phase modulation for the radial velocity and the second moment is very sensitive to non-axisymmetric pulsation components, whereas the rotational modulation of the second moment amplitude is best suited to revealing axisymmetric magnetically induced distortion of pulsations.
\end{abstract}

Key words. stars: chemically peculiar - stars: oscillations - line: profiles

\section{Introduction}

Analysis of the stellar pulsations is a valuable astrophysical technique offering a unique possibility to constrain the internal structure and evolution of stars. Detailed information about the surface geometry of stellar pulsations is a necessary prerequisite for a successful asteroseismic investigation into the physical properties of the stellar layers. Pulsational perturbations in the surface layers of stars are described by the spherical harmonic eigenfunctions. The angular degree $\ell$ and azimuthal number $m$ are sufficient to fully characterize the surface geometry of non-radial pulsations in normal slowly rotating stars.

A range of the mode identification techniques was developed to extract information about the pulsation geometry from the photometric (Watson 1988) and, most recently, spectroscopic (Aerts \& Eyers 2000; Telting 2003) observations of stellar pulsations. The moment technique, introduced by Balona (1986) and further developed by Aerts et al. (1992) and Briquet $\&$ Aerts (2003), is one of the most widely applied and efficient spectroscopic mode identification methods. It attempts to diagnose the structure of pulsational perturbations by interpreting the time variation of the first three velocity moments that are linked to the line centroid (radial velocity), line width, and skewness (line asymmetry).
In contrast to normal non-radially pulsating stars, a considerably more complex and challenging problem is posed by pulsations in rapidly oscillating Ap (roAp) stars (Kurtz \& Martinez 2000). Since their discovery (Kurtz 1982) roAp stars have drawn a lot of attention as the only example of stellar oscillations in the presence of a global strong magnetic field. This stellar magnetism is closely linked to excitation of the high-overtone short-period $p$-modes in roAp stars and, therefore, asteroseismic analysis may contribute to solving the longstanding problem of the origin and structure of global stellar magnetic fields.

The pulsational amplitude and phase of the oscillating magnetic stars were found to vary synchronously with the rotational modulation of the line of sight magnetic field. A straightforward phenomenological explanation of this behaviour was given by the oblique pulsator model (Kurtz 1982), which postulated that pulsations are aligned with the oblique magnetic field and modulation of the pulsational characteristics is a result of the periodic changes in the aspect angle at which pulsations are observed. In the standard oblique pulsator framework the modes were represented by the axisymmetric dipolar $(\ell=1, m=0)$ eigenfunction. However, both detailed photometric monitoring of some of roAp stars (e.g. Kurtz et al. 1997) and theoretical studies (Shibahashi \& Takata 1993; 
Bigot \& Dziembowski 2002; Saio \& Gautschy 2004) have suggested that pulsation modes in roAp stars are significantly distorted by rotation and magnetic field and cannot be described by a single spherical harmonic function. Recent timeresolved high-resolution spectroscopic studies of roAp stars (e.g. Kanaan \& Hatzes 1998; Kochukhov \& Ryabchikova 2001a) also revealed a startling diversity of the pulsational behaviour of individual spectral lines, which was interpreted to be an interplay between a short vertical length-scale of pulsation waves and the presence of significant vertical gradients of chemical abundance in the line-forming atmospheric regions (Kochukhov \& Ryabchikova 2001a; Ryabchikova et al. 2002).

These discoveries emphasized the necessity to develop quantitative tools to analyse the surface geometry of pulsations in roAp stars in detail in order to distinguish the horizontal and vertical pulsation effects. The measurements and modelling of the line profile moments appear especially well-suited for Ap stars which are typically very slow rotators. However, the latest version of the standard moment method implemented by Briquet \& Aerts (2003) cannot be directly applied to this kind of stars because this technique does not account for their mode obliquity and neglects magnetic distortion of pulsation modes.

The main aim of the present investigation is to develop a numerical technique suitable for modelling the moment variations of stars pulsating in distorted oblique non-radial modes. Moreover, we characterize the main features of the pulsational variability of moments in the context of modern theories of magneto-acoustic stellar oscillations. The rest of the paper is organized as follows. Section 2 discusses mathematical formalism and numerical implementation of the extended moment technique. Calculations of the moment variations are presented in Sect. 3 for a variety of oblique pulsation geometries, and Sect. 4 concludes the paper with a summary and discussion.

\section{Influence of pulsation and rotation on the line profile moments}

In the present study we use the representation of the pulsation velocity suggested by Kochukhov (2004a). This paper demonstrated that an arbitrary pulsation velocity field corresponding to a mono-periodic linear stellar oscillation with angular frequency $\omega$ can be written as

$\boldsymbol{V}(\theta, \phi, t)=\boldsymbol{V}^{\mathrm{c}}(\theta, \phi) \cos (\omega t)+\boldsymbol{V}^{\mathrm{s}}(\theta, \phi) \sin (\omega t)$,

where $\theta$ and $\phi$ are the spherical coordinates on the stellar surface, whereas $\boldsymbol{V}^{\mathrm{c}}(\theta, \phi)$ and $\boldsymbol{V}^{\mathrm{s}}(\theta, \phi)$ are the vectors describing the surface distribution of the pulsation amplitudes for the horizontal and vertical velocity fluctuations:

$\boldsymbol{V}^{\mathrm{c}}(\theta, \phi)=\left\{V_{r}^{\mathrm{c}}, V_{\theta}^{\mathrm{c}}, V_{\phi}^{\mathrm{c}}\right\}$ and $\boldsymbol{V}^{\mathrm{s}}(\theta, \phi)=\left\{V_{r}^{\mathrm{s}}, V_{\theta}^{\mathrm{s}}, V_{\phi}^{\mathrm{s}}\right\}$.

The complete expressions of the $V_{r, \theta, \phi}^{\mathrm{cs}}$ velocity components in terms of the spherical harmonics were given by Kochukhov (2004a).

In the general case of a rotating star with oblique nonradial pulsations, each oscillation mode is represented by a superposition of many spherical harmonic components
(Bigot \& Dziembowski 2002; Saio \& Gautschy 2004), and there are no coordinate transformations able to reduce this description to a single spherical harmonic eigenfunction assumed in the classical moment technique of Balona (1986) and Aerts et al. (1992). As a consequence, the six pulsation velocity components $V_{r, \theta, \phi}^{\mathrm{c}, \mathrm{s}}$ have to be defined in any coordinate system through the sums over the spherical harmonic functions $Y_{\ell m}(\theta, \phi)$ and their derivatives. Nevertheless, there still exists a special reference frame, e.g. the one connected with the global magnetic field of the star, where the pulsational displacement exhibits certain symmetries and the respective mathematical description of the velocity field is the least complicated (Saio $\&$ Gautschy 2004). We choose this coordinate system, inclined by angle $\beta$ relative to the stellar rotation axis, to define the local angular coordinates $\theta$ and $\phi$ and to specify pulsation amplitude maps $\boldsymbol{V}^{\mathrm{c}}(\theta, \phi)$ and $\boldsymbol{V}^{\mathrm{s}}(\theta, \phi)$ in terms of the sums over the spherical harmonic components.

For the purpose of numerical computing the disk-averaged characteristics of the line of sight velocity, the visible stellar hemisphere is divided into a number of surface zones with approximately equal areas (as in Piskunov \& Kochukhov 2002). Position of each surface element in the stellar reference frame is characterized by the latitude $\rho(-\pi / 2 \leq \rho \leq \pi / 2)$ and the longitude $l(0 \leq l \leq 2 \pi)$.

The transformation of an arbitrary vector field from the spherical coordinate system not aligned with the stellar rotation axis to the Descartes reference frame of the observer was treated by Piskunov \& Kochukhov (2002) in the context of modelling the stellar magnetic field. Similar expressions are applicable to the pulsation velocity field. The transformation of the vectors $\boldsymbol{V}^{\mathrm{c}}$ and $\boldsymbol{V}^{\mathrm{s}}$ can be written as a series of matrix multiplications employing the operator $\mathrm{T}(\gamma, \delta)$ defined as

$\mathrm{T}(\gamma, \delta) \equiv\left(\begin{array}{ccc}\cos \delta & -\sin \delta & 0 \\ \sin \delta & \cos \delta & 0 \\ 0 & 0 & 1\end{array}\right) \times\left(\begin{array}{ccc}1 & 0 & 0 \\ 0 & \cos \gamma & -\sin \gamma \\ 0 & \sin \gamma & \cos \gamma\end{array}\right)$,

where angle $\gamma$ describes a tilt of the polar $(z)$ axis of a given coordinate system and angle $\delta$ corresponds to a rotation around this axis. Thus, vector components of the pulsation velocity amplitudes in the observer's coordinate system whose $z$-axis is directed along the line of sight are evaluated as

$\left(\begin{array}{r}V_{x}^{\mathrm{c}, \mathrm{s}}(\rho, l) \\ V_{y}^{\mathrm{c}, \mathrm{s}}(\rho, l) \\ -V_{z}^{\mathrm{c}, \mathrm{s}}(\rho, l)\end{array}\right)=\mathrm{T}(-i, 0) \mathrm{T}(\beta, \varphi) \mathrm{T}\left(\theta-\frac{\pi}{2}, \phi\right)\left(\begin{array}{r}V_{\phi}^{\mathrm{c}, \mathrm{s}}(\theta, \phi) \\ -V_{r}^{\mathrm{c}, \mathrm{s}}(\theta, \phi) \\ -V_{\theta}^{\mathrm{c}, \mathrm{s}}(\theta, \phi)\end{array}\right)$.

Here $\varphi \equiv \Omega t$ is the phase angle corresponding to uniform stellar rotation with the angular frequency $\Omega$ and $i$ is the angle between the stellar rotation axis and the line of sight.

The total time-dependent velocity with respect to the observer includes contributions from pulsation and rotation:

$V_{z}(\rho, l)=V_{z}^{\mathrm{c}}(\rho, l) \cos (\omega t)+V_{z}^{\mathrm{s}}(\rho, l) \sin (\omega t)+V_{z}^{\mathrm{rot}}(\rho, l)$.

The $z$ component of the rotation velocity is given by

$V_{z}^{\mathrm{rot}}(\rho, l)=\cos \rho \sin (l+\varphi) v_{\mathrm{e}} \sin i$,

where $v_{\mathrm{e}} \sin i$ is the projected rotational velocity. 
We use the usual assumption that the intrinsic line profile can be approximated by a Gaussian with variance $\sigma^{2}$. This approximation will fail only for a (relatively uncommon) situation of a magnetically sensitive line in the spectrum of a strongly magnetic and very slowly rotating roAp star. We follow the moment definitions of Aerts et al. (1992) and express variability of moments in terms of changes with pulsation frequency $\omega$, as well as its first and second harmonics, $2 \omega$ and $3 \omega$. The expressions for the radial velocity $\langle V\rangle$, the second moment $\left\langle V^{2}\right\rangle$, and the third moment $\left\langle V^{3}\right\rangle$ thus become

$$
\begin{aligned}
\langle V\rangle=\langle & \left.V_{z}(\rho, l)\right\rangle=\bar{V}_{100} \cos (\omega t)+\bar{V}_{010} \sin (\omega t), \\
\left\langle V^{2}\right\rangle= & \sigma^{2}+\left\langle V_{z}^{2}(\rho, l)\right\rangle=\sigma^{2}+\frac{1}{2}\left(\bar{V}_{200}+\bar{V}_{020}+2 \bar{V}_{002}\right) \\
& +2 \bar{V}_{101} \cos (\omega t)+2 \bar{V}_{011} \sin (\omega t) \\
& +\frac{1}{2}\left(\bar{V}_{200}-\bar{V}_{020}\right) \cos (2 \omega t)+\bar{V}_{110} \sin (2 \omega t)
\end{aligned}
$$

and

$$
\begin{aligned}
\left\langle V^{3}\right\rangle= & \left\langle V_{z}^{3}(\rho, l)\right\rangle+3 \sigma^{2}\left\langle V_{z}(\rho, l)\right\rangle=\frac{3}{2}\left(\bar{V}_{201}+\bar{V}_{021}\right) \\
& +\frac{3}{4}\left(\bar{V}_{300}+4 \bar{V}_{102}+\bar{V}_{120}+4 \sigma^{2} \bar{V}_{100}\right) \cos (\omega t) \\
& +\frac{3}{4}\left(\bar{V}_{030}+4 \bar{V}_{012}+\bar{V}_{210}+4 \sigma^{2} \bar{V}_{010}\right) \sin (\omega t) \\
& +\frac{3}{2}\left(\bar{V}_{201}-\bar{V}_{021}\right) \cos (2 \omega t)+3 \bar{V}_{111} \sin (2 \omega t) \\
& +\frac{1}{4}\left(\bar{V}_{300}-3 \bar{V}_{120}\right) \cos (3 \omega t) \\
& +\frac{1}{4}\left(3 \bar{V}_{210}-\bar{V}_{030}\right) \sin (3 \omega t) .
\end{aligned}
$$

The quantities $\bar{V}_{k n m}$ represent the surface integrals of various powers of the $V_{z}^{\mathrm{c}}, V_{z}^{\mathrm{s}}$, and $V_{z}^{\text {rot }}$ velocity components and are approximated with the weighted sums over the visible surface elements

$\bar{V}_{k n j}(\varphi) \equiv \sum_{i=1}^{N} W_{i}\left[V_{z}^{\mathrm{c}}(\rho, l)\right]^{k}\left[V_{z}^{\mathrm{s}}(\rho, l)\right]^{n}\left[V_{z}^{\mathrm{rot}}(\rho, l)\right]^{j}$

Here the weight function $W_{i}$ is evaluated under the assumption of the linear limb-darkening law

$W_{i} \equiv \frac{3\left(1-u+u \mu_{i}\right) \mu_{i} S_{i}}{\pi(3-u)}$,

where $u$ is the limb-darkening coefficient, whereas $\mu_{i}$ and $S_{i}$ are the cosine between the surface normal and the line of sight and the intrinsic area of the $i$ th surface zone, respectively. In deriving expressions (7)-(9) we took the symmetry of the rotation velocity field into account, which reduces the $\bar{V}_{00 j}$ sums to zero for all odd values of $j$.

We emphasize that the moment Eqs. (7)-(9) include variability on the two different timescales: pulsation with the angular frequency $\omega$ and rotation with the angular frequency $\Omega$. The latter is included in the expressions indirectly through the time dependence of the coordinate transformation defined by Eq. (4). Taking this fundamental background of the problem of investigation into the nature of oblique non-radial pulsators into account it is natural to take advantage of stellar rotation and to observe the pulsation velocity field at different aspect angles - a unique possibility realized only with roAp stars. Then the mode identification for roAp stars and the analysis of the distortion of pulsations by rotation and magnetic field can be carried out by modelling the temporal evolution of the pulsation spectra of $\langle V\rangle,\left\langle V^{2}\right\rangle$, and $\left\langle V^{3}\right\rangle$ as a function of the stellar rotation phase, similar to the studies of the rapid photometric variability of roAp stars (Kurtz \& Martinez 2000). To describe the theoretical rotational modulation of each amplitude $V_{\mathrm{x}}(\varphi)$ in Eqs. (7)-(9) we compute an equivalent representation in terms of the total time-dependent amplitude $A_{\mathrm{x}}(\varphi)$ and phase $\Phi_{\mathrm{x}}(\varphi)$

$$
\begin{aligned}
V_{\mathrm{x}}(t) & =a_{\mathrm{x}}(\varphi) \cos (n \omega t)+b_{\mathrm{x}}(\varphi) \sin (n \omega t) \\
& =A_{\mathrm{x}}(\varphi) \cos \left[n \omega t+\Phi_{\mathrm{x}}(\varphi)\right],
\end{aligned}
$$

where

$A_{\mathrm{x}}(\varphi)=\sqrt{a_{\mathrm{x}}^{2}(\varphi)+b_{\mathrm{x}}^{2}(\varphi)}$ and $\Phi_{\mathrm{x}}(\varphi)=\arctan \frac{a(\varphi)}{b(\varphi)}-\frac{\pi}{2}$.

Examination of the moment formulae derived for the oblique pulsators reveals some essential differences with respect to the moment behaviour in normal pulsating stars. Apart from the expected rotational modulation of all terms in the moment amplitudes and phases, the most important feature is the relative amplitudes of the terms contributing to the pulsational variation of the second moment. In stars where pulsations are aligned with the rotation axis and the pulsation velocity is on the same order as the rotation one, contribution of the main frequency term is usually small or comparable to the $2 \omega$ term and even reduces to zero for any rotation velocity, if pulsations are axisymmetric (i.e., in the language of terms of Eq. (8): $\left.\bar{V}_{101,011}(\beta=0, m=0)=0\right)$. This does not happen for oblique pulsators. For all but the slowest rotating roAp stars pulsation amplitudes are smaller than the projected rotational velocity. Hence, $\bar{V}_{101,011}(\beta \neq 0) \gg\left(\bar{V}_{200}-\bar{V}_{020}\right), \bar{V}_{110}$ even for an axisymmetric pulsation and we expect to see a strong single-wave variability dominating the pulsation behaviour of $\left\langle V^{2}\right\rangle$ in most of the roAp stars.

Another interesting feature of the extended moment equations is rotational modulation of the "constant" terms in the expression for the second moment and the presence of such terms for the third moment. Although changes of these terms on the timescale of stellar rotation are relatively small, this effect nicely illustrates a consequence of non-asymmetric coaddition of both pulsation and rotation velocity fields and may contribute to line profile variation in the regular (averaged over many pulsation cycles) spectroscopic observations of roAp stars.

In comparison to the classical semi-analytical expressions of the moment variation derived by Aerts et al. (1992), the numerical scheme introduced here is considerably more general and straightforward to implement. It is also more flexible due to the possibility of including additional physical effects (such as stellar surface inhomogeneities) in the weight function $W$. A similar numerical approach was also followed in a recent modification of the moment method presented by Briquet \& Aerts (2003), who optimized the moment technique for multiperiodic rotating stars but did not address the problem of modelling the moment variation for stars with oblique pulsations. 

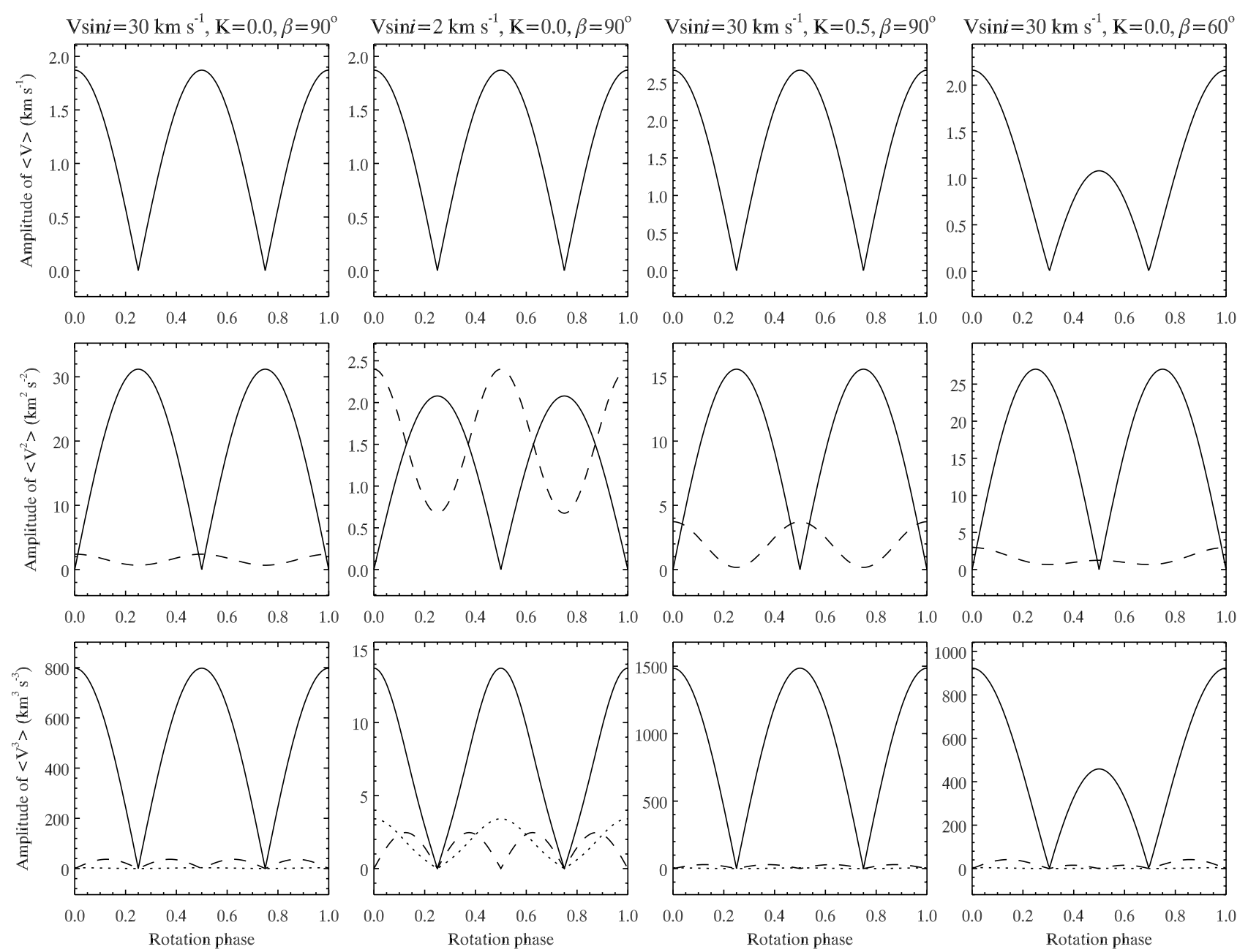

Fig. 1. Rotational modulation of the pulsation amplitudes for the radial velocity $\langle V\rangle$, second moment $\left\langle V^{2}\right\rangle$, and third moment $\left\langle V^{3}\right\rangle$ computed for the oblique dipolar $(\ell=1, m=0)$ non-radial mode with $V_{\max }=4 \mathrm{~km} \mathrm{~s}^{-1}$ and $i=60^{\circ}$. The other relevant parameters - the projected rotation velocity $v_{\mathrm{e}} \sin i$, the ratio of the horizontal to vertical pulsation amplitude $K$, and the mode obliquity $\beta$ - are indicated at the top of the uppermost panel in each column of plots. Different curves show amplitudes for the terms changing with pulsation frequency (solid line), its first (dashed line) and second (dotted line) harmonics.

\section{Illustration of the moment variations}

For all calculations of the line profile moments presented in this section we used a 1876-element surface grid and linear limbdarkening coefficient $u=0.5$, and adopted an intrinsic profile with $\sigma=5 \mathrm{~km} \mathrm{~s}^{-1}$. The maximum surface amplitude of the pulsation velocity was set to $V_{\max }=\max \left(\sqrt{\left(V^{\mathrm{c}}\right)^{2}+\left(V^{\mathrm{s}}\right)^{2}}\right)=$ $4 \mathrm{~km} \mathrm{~s}^{-1}$. These values correspond to a typical limb-darkening, thermal Doppler line width, and pulsational amplitude of the late-A rapidly oscillating peculiar stars. The reference values of the other relevant parameters were chosen to be close to those of the prototype roAp star HR 3831 (Kochukhov et al. 2004; Kochukhov 2004b): $v_{\mathrm{e}} \sin i=30 \mathrm{~km} \mathrm{~s}^{-1}, i=60^{\circ}, \beta=90^{\circ}$. We also attempt to test an effect of a significant horizontal pulsation component by comparing calculations with the ratio of the horizontal to vertical pulsation amplitude $K=0.5$ (cf. Saio \& Gautschy 2004) with the case of purely vertical pulsations.

Figures 1-3 present rotational modulation of the amplitude and phase of each term in the variation of the first three moments.

\subsection{Axisymmetric dipolar modes}

The classical oblique pulsator model is represented by a pure axisymmetric dipolar $(\ell=1, m=0)$ mode inclined with respect to the rotation axis (Kurtz 1982). If the mode obliquity is close to $90^{\circ}$, the radial velocity variation reaches maximum at the two phases during rotation cycle (Fig. 1), corresponding to the moments of the best visibility of each of the two pulsation poles. The amplitude modulation is clearly non-sinusoidal, as it features sharp minima and broad maxima.

In agreement with the qualitative discussion in Sect. 2, in the case of a moderately rapid rotation $\left(v_{\mathrm{e}} \sin i \gg V_{\max }\right)$, variation of $\left\langle V^{2}\right\rangle$ is dominated by the main frequency term and occurs with the $\pi / 2$ radian shift in rotation phase relative to the modulation of radial velocity. As demonstrated by Fig. 1, variation with twice the pulsation frequency becomes dominant only when the maximum pulsation velocity significantly exceeds $v_{\mathrm{e}} \sin i$. Modulation of the respective term in the amplitude of the second moment is sinusoidal and occurs out of phase with respect to modulation of the main frequency term. 

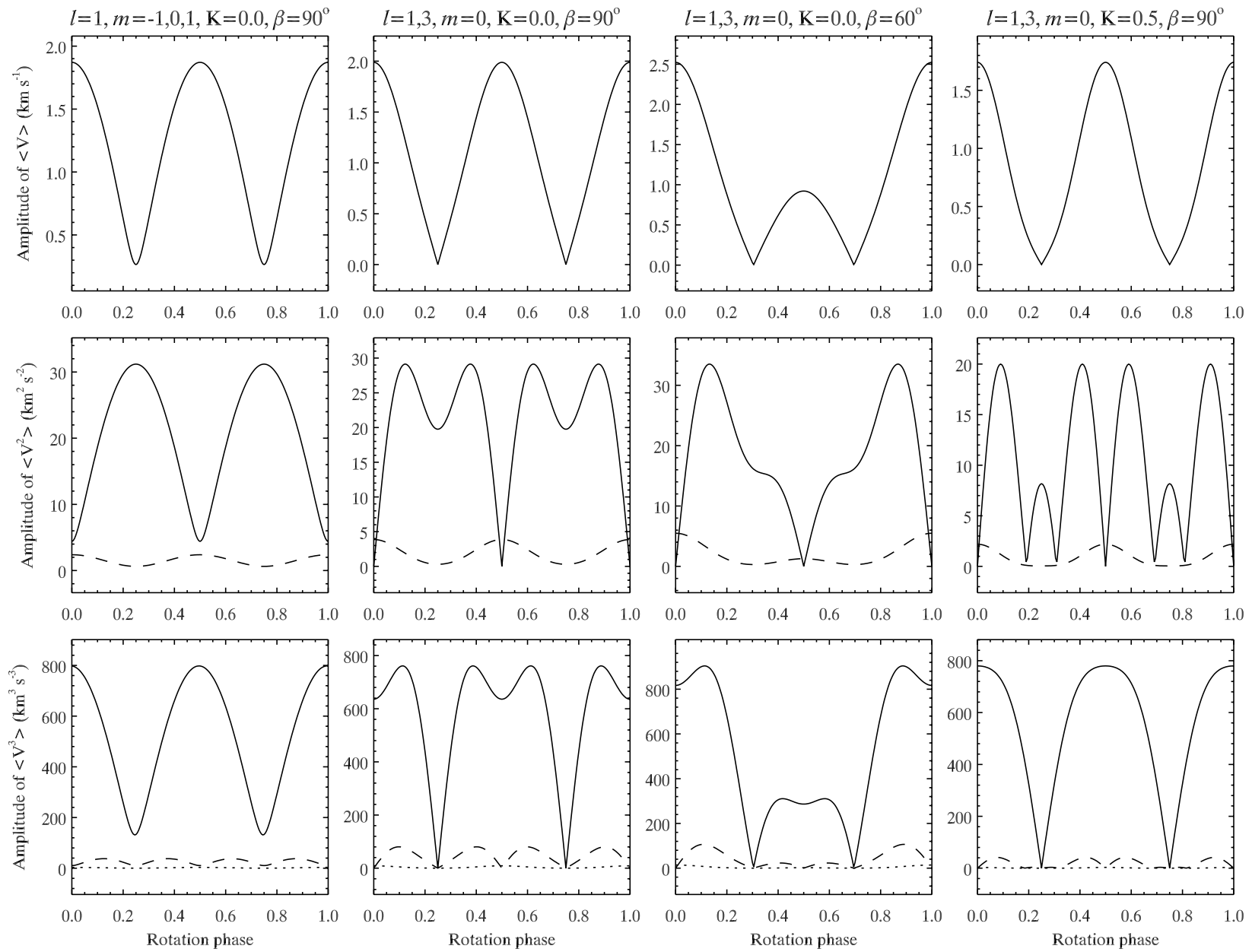

Fig. 2. Same as Fig. 1 for the amplitude modulation of the moments computed for distorted dipolar modes. First column: non-axisymmetric dipolar mode with $V_{m=1}=V_{m=-1}=0.1 V_{m=0}$; second to fourth columns: magnetically distorted axisymmetric mode with $V_{\ell=3}=0.5 V_{\ell=1}$ and different $K$ and $\beta$.

Harmonic contributions to the pulsational changes of the third moment are always small.

A significant horizontal pulsation displacement with the ratio of the horizontal to vertical pulsation amplitude $K=0.5$ has no effect on the shape of the rotational modulation of the amplitudes of moments. At the same time, horizontal motions have a profound effect on the maximum amplitude achieved during rotation cycle, as the $\left\langle V^{2}\right\rangle$ amplitude decreases by roughly a factor of 2 , whereas the maximum amplitude of the third moment increases by the same amount.

For an intermediate $\left(\beta=60^{\circ}\right)$ obliquity of the axisymmetric dipolar mode we preferentially sample one of the pulsation poles. Consequently, one of the maxima of the amplitude of $\langle V\rangle$ and $\left\langle V^{3}\right\rangle$ decreases. The rotational modulation of the amplitude of the second moment is weakly affected.

The pulsational phase modulation of the axisymmetric oblique dipolar modes is illustrated in the first and second columns of Fig. 3. The appearance of the phase modulation curves does not depend on $v_{\mathrm{e}} \sin i$ and the presence of the horizontal pulsation fluctuations. The phase of radial velocity exhibits two $\pi$ radian phase jumps characteristic of the oblique dipolar pulsation. The rapid change in phase occurs when pulsation equator is passing through the plane defined by the rotation axis and the line of sight. In contrast, the $\pi$ radian jump in the phase of the main frequency term of the second moment occurs when pulsation poles are facing the observer. The phase of the first harmonic term is not modulated by the stellar rotation, whereas the respective term in the pulsational changes of the third moment shows four jumps during each rotation cycle.

An effect of the moderate mode obliquity on the phase modulation is illustrated in the second column of Fig. 3. A decrease in the visibility of one of the pulsation poles does not change the overall character of the phase modulation, but modifies the times of the $\pi$ radian jumps, so the latter are no longer equidistant in rotation phase.

\subsection{Non-axisymmetric dipolar modes}

In their recent theoretical study Bigot \& Dziembowski (2002) have introduced a modified oblique pulsator model of roAp stars, which represented a significant departure from the classical axisymmetric dipolar pulsation geometry. Bigot \& Dziembowski (2002) suggested that an accurate nonperturbative treatment of the interaction between stellar rotation, pulsations, and magnetic field may lead to representation of the pulsation eigenmodes with a complex superposition of 

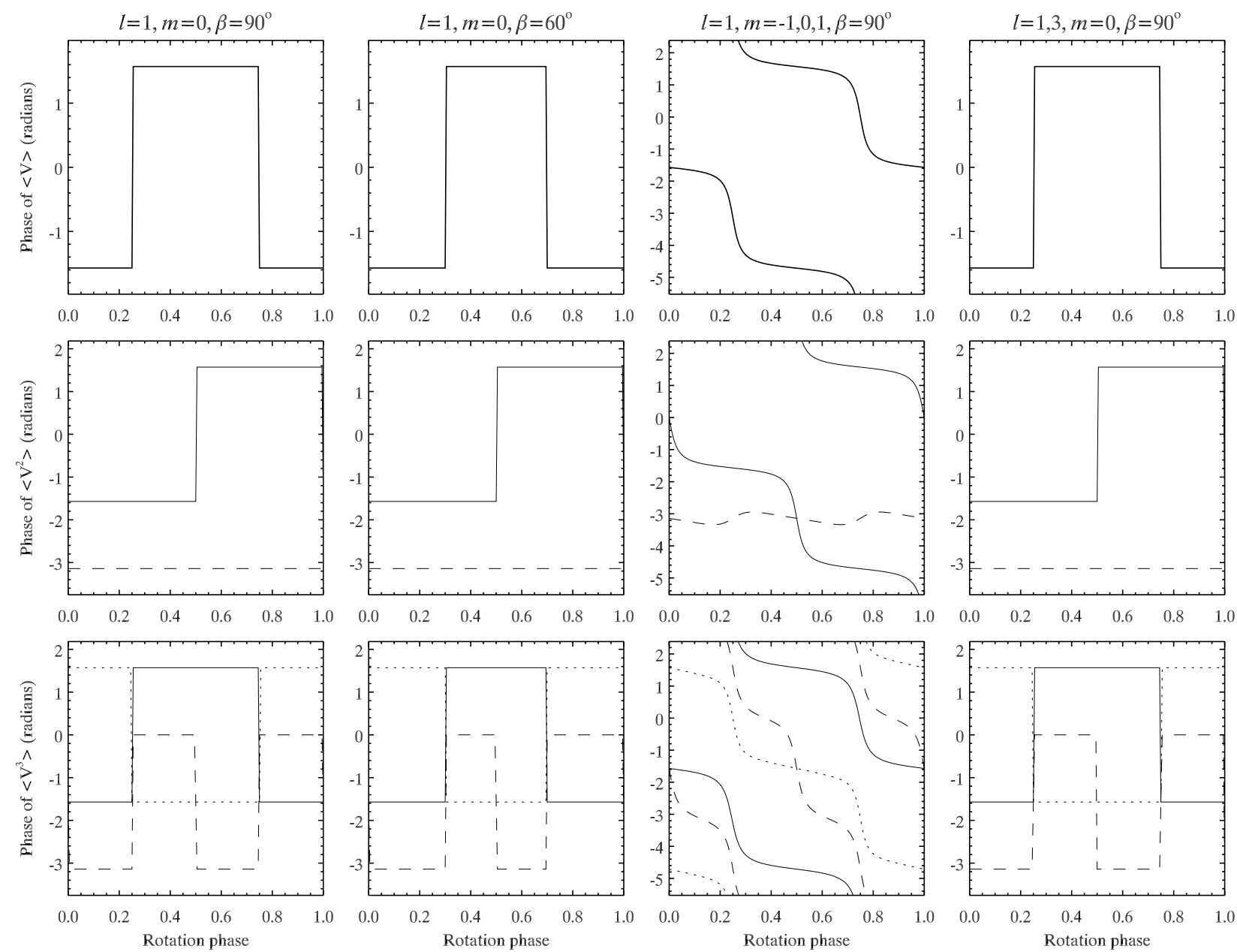

Fig. 3. Rotational modulation of the pulsation phase of the line profile moments. The format of the figure is similar to that of Figs. 1 and 2.

the spherical harmonic functions that contain substantial nonaxisymmetric components. Furthermore, strong effects due to the centrifugal force are expected to break locking of the pulsation axis with the dipolar magnetic field, so that pulsations are aligned with neither the magnetic nor rotation axes. However, this entirely new geometrical picture of the roAp oscillations remains somewhat speculative, as Bigot \& Dziembowski (2002) gave no general predictions for the amplitude of the non-axisymmetric contribution to the pulsation velocity field and failed to consider distortion of the pulsation modes due to a strong magnetic field typical for the majority of roAp stars.

It turns out that analysis of the moment variation provides a very sensitive diagnostic of non-axisymmetric pulsation. In this section we investigate an effect of small non-axisymmetric ( $m= \pm 1$ ) contributions to the dominant oblique dipolar mode. In the pulsation reference frame the amplitude of the nonaxisymmetric components is set to $10 \%$ of the velocity due to the $\ell=1, m=0$ component. This distortion of the dipolar pulsation has a marginal effect on the modulation of the amplitudes of moments (Fig. 2, first column). Rotational changes of the radial velocity amplitude become smoother and the amplitude does not drop to zero at phases 0.25 and 0.75 . At the same time, even very small non-axisymmetric components have a dramatic effect on the phase modulation curves, as illustrated in the third column of Fig. 3. The $\pi$ radian jumps vanish and are replaced by the steep but continuous phase changes. This phenomenon is a straightforward consequence of the surface distribution of the pulsation velocity phase. All axisymmetric modes have constant phase with the $\pi$ radian jumps at the node lines, whereas the non-axisymmetric pulsation components are characterized by a continuous change of the pulsation phase from one surface zone to the next. This is readily reflected in the disk-averaged velocity moments and allows identification of a very low amplitude non-axisymmetric component of oblique pulsations.

\subsection{Magnetically distorted dipolar modes}

In another important theoretical development Saio \& Gautschy (2004) have studied the effect of a strong magnetic field on the geometry of oblique non-radial modes. The stellar rotation and resulting non-axisymmetric pulsational contributions were neglected, but calculations were performed for the magnetic field strength of up to $9 \mathrm{kG}$. In the oblique pulsator model of Saio \& Gautschy (2004) pulsations remain axisymmetric and are aligned with the magnetic field axis. The effect of dipolar magnetic field is to constrain oscillations to the surface areas close to magnetic poles. Thus, an initially dipolar pulsation is distorted in such a way that it is described by a sum of the 
odd axisymmetric pulsation components. We computed moment variation for such a pulsation model assuming that the octupolar, $\ell=3$, pulsation has a $50 \%$ smaller amplitude compared to the $\ell=1$ component and contribution of other harmonics is negligible.

The amplitude modulation of the line profile moments computed for magnetically distorted oblique pulsations is presented in Fig. 2 (Cols. 2-4). The most significant effect of the $\ell=3$ component appears in the modulation of the second moment amplitude. The respective curve becomes more complex and two additional minima develop at the rotation phases 0.25 and 0.75 . Variation of the second moment is also quite sensitive to the obliquity of the mode and to the presence of the horizontal pulsation motions (predicted in the theory of Saio \& Gautschy 2004). Thus, magnetic distortion and related enhancement of the horizontal amplitude of pulsations in roAp stars can be most efficiently studied along with the analysis of the rotational modulation of the variation of the second moment of spectral line profiles.

Despite a strong influence on the moment amplitudes, a moderate contribution of the octupolar axisymmetric spherical harmonic considered here does not change modulation of the phase curves (Fig. 3, fourth column); instead it remains identical to the phase modulation obtained for the pure oblique dipolar mode.

\section{Summary and discussion}

Recent advances in theoretical studies of the stellar magnetoacoustic pulsations emphasize a need to obtain detailed empirical information about the structure of the pulsational disturbances in the atmospheres of roAp stars. Until recently, analyses of this interesting class of non-radial pulsators were limited to time-resolved photometric observations. Interpretation of the broad-band photometric variability turned out to be a challenging task because of the difficulty of discriminating between various effects that contribute to the luminosity variation. Anomalous wavelength dependence of the photometric pulsation amplitudes and phases (Watson 1988), as well as non-linearity and non-adiabatic effects (Medupe 2002) are also not understood. As a result of these complications, little progress has been made in understanding the vertical and horizontal geometry of the roAp pulsations beyond the generic oblique pulsator model of Kurtz (1982). In fact, the recent study by Saio \& Gautschy (2004) has demonstrated that, due to the averaging over the visible stellar hemisphere, the photometric observables are very weakly sensitive to the details of the pulsation geometry and turn out to be practically useless in testing predictions of elaborate theoretical models.

The newly emerged field that observes and interprets the pulsational line profile variations in roAp stars (Kochukhov \& Ryabchikova 2001a) offers a more promising research direction. Due to their simple relation to pulsational displacement, the spectroscopic observables provide a more direct, reliable, and general approach to modelling the roAp pulsations compared to interpreting the photometric pulsational data, which requires some restrictive assumptions about the physics of pulsations. The most detailed and straightforward way to reveal the pulsation geometry of roAp stars is to construct a twodimensional image of the pulsation velocity field from the line profile variation using the pulsation Doppler imaging technique (Kochukhov 2004a,b). However, this sophisticated modelling requires spectroscopic observational data of a superb quality and is limited to moderately rotating roAp stars. An alternative possibility is to apply the moment method (Briquet \& Aerts 2003), which is based on interpretation of the low-order moments of the absorption line profiles and can be applied to very slowly rotating stars.

In this paper we have investigated the diagnostic potential of the moment method in its application to the oblique distorted stellar pulsations. We extended the moment technique to deal with the stellar pulsation velocity field with a symmetry axis other than the axis of stellar rotation and developed a numerical method for computing the rotational modulation of amplitudes and phases of different terms in the variation of the line profile moments. Calculations with our new version of the moment technique reveal some important differences in the behaviour of the line profile moments in normal and oblique pulsators. In particular, for all but the slowest rotating roAp stars with slightly distorted dipolar modes we expect to observe a single-wave variation of $\left\langle V^{2}\right\rangle$ (the second moment) during the pulsation cycle ${ }^{1}$. Furthermore, a non-axisymmetric superposition of the pulsation and rotation velocity distributions leads to new terms in the variation of the higher line profile moments, which are modulated by the stellar rotation but are constant on the pulsation timescale.

Calculation of the line profile moments for various pulsation geometries of roAp stars predicted by the theoretical studies demonstrates that analyzing the rotational modulation of variability of the first two moments $\left(\langle V\rangle\right.$ and $\left.\left\langle V^{2}\right\rangle\right)$ is sufficient for diagnosing various non-axisymmetric and axisymmetric distortions of the basic oblique dipolar pulsation geometry and allows us to carry out an extremely sensitive verification of the theories attempting to describe an interaction between pulsation, magnetic field, and stellar rotation. For instance, the non-axisymmetric modified oblique pulsator theory of Bigot \& Dziembowski (2002) can be very efficiently tested for a large number of roAp stars by using observations of the rotational modulation of the pulsation phase of radial velocity and higher line profile moments. The presence of a smooth phase modulation instead of the $\pi$ radian jumps is a unique signature of non-axisymmetric modes. It reveals a weak non-axisymmetric contribution even in the background of a much stronger axisymmetric component. Similarly, the presence of the odd axisymmetric harmonic components arising due to the influence of the global dipolar magnetic field (Saio \& Gautschy 2004) can be verified by observation of the second moment variation.

The promising diagnostic potential of this new moment technique can be realised through interpretation of the high resolution observations of the line profile variation in oblique pulsators. The bright, high-amplitude roAp stars with short

1 The presence of such single-wave second moment variation in the extremely slowly rotating roAp star $\gamma$ Equ (Kochukhov \& Ryabchikova 2001a) is thus confirmed to be a signature of a nonaxisymmetric non-dipolar pulsation. 
rotation periods $\left(\lesssim 10^{\mathrm{d}}\right)$ are the best candidates for application of the moment method, because their surface pulsation velocity structure can be observed from different aspect angles during an observing run of a few nights. Observational data for the prototype roAp star HR 3831 have already been acquired by Kochukhov \& Ryabchikova (2001b). The time-series analysis of this object and interpretation of the pulsational spectral variation with the moment technique will be presented in a forthcoming paper.

Acknowledgements. This work was partially supported by the postdoctoral stipend from the Swedish Research Fund and by the Lise Meitner fellowship from the Austrian Science Fund (FWF, project No. M757-N02).

\section{References}

Aerts, C., De Pauw, M., \& Waelkens, C. 1992, A\&A, 266, 294

Aerts, C., \& Eyer L. 2000, in Delta Scuti and Related Stars, ed. M. Breger, \& M. Montgomery, ASP Conf. Ser., 210, 113
Balona, L. A. 1986, MNRAS, 219, 111

Bigot, L., \& Dziembowski, W. A. 2002, A\&A, 391, 235

Briquet, M., \& Aerts, C. 2003, A\&A, 398, 687

Kanaan, A., \& Hatzes, A. P. 1998, ApJ, 503, 848

Kochukhov, O. 2004a, A\&A, 423, 613

Kochukhov, O. 2004b, ApJ, 615, L149

Kochukhov, O., Drake, N. A., Piskunov, N., \& de la Reza, R. 2004, A\&A, 424, 935

Kochukhov, O., \& Ryabchikova, T. 2001a, A\&A, 374, 615

Kochukhov, O., \& Ryabchikova, T. 2001b, A\&A, 377, L22

Kurtz, D. W. 1982, MNRAS, 200, 807

Kurtz, D. W., \& Martinez, P. 2000, Baltic Astron., 9, 253

Kurtz, D. W., van Wyk, F., Roberts, G., et al. 1997, MNRAS, 287, 69

Medupe, R. 2002, Ph.D. Thesis, University of Cape Town

Piskunov, N., \& Kochukhov, O. 2002, A\&A, 381, 736

Ryabchikova, T., Piskunov, N., Kochukhov, O., et al. 2002, A\&A, 384, 545

Saio, H., \& Gautschy, A. 2004, MNRAS, 350, 485

Shibahashi, H., \& Takata, M. 1993, PASJ, 45, 617

Telting, J. 2003, Ap\&SS, 284, 85

Watson, R. D. 1988, Ap\&SS, 140, 255 